\title{
Correction to: Dual-Purpose Bioreactors to Monitor Noninvasive Physical and Biochemical Markers of Kidney and Liver Scaffold Recellularization, by Uzarski JS, Bijonowski BM, Wang $\mathrm{B}$, Ward $\mathrm{HH}$, Wandinger-Ness A, Miller WM, and Wertheim JA. Tissue Eng Part C Methods 2015;21(10):1032-1043. DOI: 10.1089/ten.tec.2014.0665
}

$\mathbf{T}$ He ARTICle entitled, Dual-Purpose Bioreactors to Monitor Noninvasive Physical and Biochemical Markers of Kidney and Liver Scaffold Recellularization, by Uzarski JS, Bijonowski BM, Wang B, Ward HH, Wandinger-Ness A, Miller WM, and Wertheim JA. Tissue Eng Part C Methods. October 2015, 21(10): 1032-1043. DOI: 10.1089/ten.tec.2014.0665. The article erroneously reported the cells used in the study were human instead of canine. The following explanation for the discrepancies was provided by the corresponding author of the article, Jason A. Wertheim, MD, PhD.

The human RCTEC/RCTE cell stocks reported to be used here were originally provided by Dr. Loghman-Adham (then at St. Louis University) to co-author Dr. Wandinger-Ness (University of New Mexico) through an MTA in 2001. The SV40 immortalized human RCTEC/RCTE cells were characterized by Loghman-Adham et al. (Renal Physiology, 285, F397, 2003) as being a distal renal tubule cell line. We use routine short tandem repeat (STR) DNA sequencing (Performed by IDEXX BioResearch) to authenticate key biological resources, as encouraged by the US National Institutes of Health. When the earliest passages of the RCTEC/RCTE cell stocks available in the Wandinger-Ness laboratory (passage 18) were interrogated, this analysis indicated cells were of mixed lineage. Further analyses of PCR products using QIAxcel capillary electrophoresis and sequencing demonstrated the presence of a canine product. A set of canine specific STR markers were compared to the sample and showed that population had a genetic profile with $92 \%$ identity to the Madin-Darby Canine Kidney (MDCK) cell line. Later cell stocks that were used in the present publication were STR profiled and showed drift to $100 \%$ MDCK lineage. Despite being of canine origin, rather than human as was previously thought, MDCK is similarly a distal renal tubule epithelial cell line, and for purposes of rigor, reproducibility and experimental validation by others we report on this misidentification and have updated the online version of the manuscript accordingly. This information will also be reported for listing on the International Cell Line Authentication Committee (ICLAC) database (http://iclac.org).

The changes made in the online article are listed below and shown in bold:

Page 1032, in the $12^{\text {th }}$ line from the beginning of the Abstract, the phrase "model human cells are introduced," should be changed to "model parenchymal cells are introduced".

Page 1035, following the subheads Recellularization/Kidney scaffolds is the phrase "Model human cells are used". It should be changed to "Model parenchymal cells are used".

Page 1035, in the 3rd line below the subheads Recellularization/Kidney scaffolds is the phrase "ECM components and human parenchymal cells." The word human should be deleted so the phrase reads, "ECM components and parenchymal cells".

Page 1035, in the right column, in the $13^{\text {th }}$ and $14^{\text {th }}$ lines the phrase "immortalized human renal cortical tubular epithelial (RCTE)

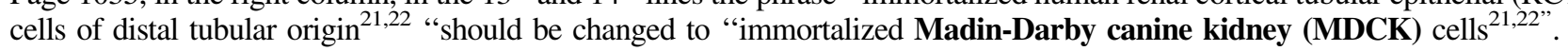

Page 1036, in the left column, in the $3^{\text {rd }}$ and $4^{\text {th }}$ lines from the top of the column, the phrase "RCTE cells are a wellestablished immortalized line of human distal renal tubule cells". The phrase should be changed to "MDCK cells are a well-established immortalized line of renal epithelial cells." 
Page 1038, in the left column, in the $11^{\text {th }}$ line from the top of the column, the phrase "RCTE cells were injected" should be changed to "MDCK cells were injected".

Page 1038, in the left column, in the $2^{\text {nd }}$ line from the bottom of the column the phrase, "RCTE cells" should be changed to "MDCK cells".

Page 1038, in the right column, in the $6^{\text {th }}$ line from the top of the column, "RCTE cells" should be changed to "MDCK cells".

Page 1038, in the right column, in the $5^{\text {th }}$ line from the bottom of the column, "RCTE cells" should be changed to "MDCK cells".

Page 1039 , in the left column, in the $2^{\text {nd }}$ line from the top of the column, "RCTE cells" should be changed to "MDCK cells".

Page 1041, in the right column, on the $13^{\text {th }}$ line below the subhead "Conclusions," the phrase "xenogeneic model human cell lines" should be changed to "xenogeneic model cell lines". The word human is deleted.

Page 1042, at the end of reference 13, the following sentence was added, "* RCTE cells referenced in this publication were confirmed to be MDCK cells."

Page 1042, references 21 and 22 have been replaced with:

21. Hall, H.G., Farson, D.A., and Bissell, M.J. Lumen formation by epithelial cell lines in response to collagen overlay: a morphogenetic model in culture. Proc Natl Acad Sci U S A 79, 4672, 1982.

22. McAteer, J.A., Evan, A.P., and Gardner K.D., Morphogenetic clonal growth of kidney epithelial cell line MDCK. Anat Rec 217, 229, 1987.

The authors apologize for the misidentification. 